\title{
Tigecycline Therapy for Multi-drug-Resistant Pseudomonas aeruginosa Sepsis Associated with Multi-organ Failure in an Infant with Persistent Arterial Duct. Case Report
}

\author{
Veronika Krasnanova $^{1}$ (D) $\cdot$ Lubica Kovacikova $^{1}$
}

Accepted: 6 July 2020 / Published online: 13 July 2020

(C) Springer Nature Switzerland AG 2020

\begin{abstract}
Sepsis is the leading cause of death in infants and children worldwide. The growing drug resistance in nosocomial gram-negative bacteria has resulted in treatment challenges. One of the most common multi-drug-resistant bacteria is Pseudomonas aeruginosa. Resistance to antibiotics used in Pseudomonas aeruginosa infections limits the therapeutic options. We present a tigecycline administration in a 5-month-old infant with patent arterial duct, heart failure, and respiratory failure due to respiratory syncytial virus bronchiolitis with subsequent respiratory distress syndrome and severe sepsis caused by multi-drug-resistant Pseudomonas aeruginosa. Despite combined antibiotic therapy with meropenem, amikacin, and colistin, inflammatory markers increased. Because of life-threatening condition, tigecycline was added to the therapy and was administered intravenously twice daily. Within $48 \mathrm{~h}$, inflammatory markers started to decrease and tigecycline therapy continued for 13 days without adverse effects. Tigecycline used in combination with other antibiotics might be a valuable therapeutic approach in the management of multidrug-resistant bacteria infections in pediatric patients when conventional antibiotics have failed. Further studies are needed to evaluate the efficacy and safety of tigecycline administration in critically ill pediatric patients.
\end{abstract}

Keywords Sepsis $\cdot$ Tigecycline $\cdot$ Infant $\cdot$ Multi-drug-resistant Pseudomonas aeruginosa

\section{Introduction}

Severe sepsis in critically ill infant in the intensive care unit is a major healthcare problem associated with high morbidity and mortality. In recent years the growing drug resistance in nosocomial gram-negative bacteria has resulted in treatment challenges for physicians, mostly for pediatricians $[1,2]$. Despite the rising relevance of Pseudomonas aeruginosa infections in adults, only a few studies have evaluated this condition in pediatric population [3, 4]. This case report describes the efficacy and safety of glycylcycline antibiotic tigecycline as a salvage therapy for an infant with severe sepsis caused by multi-drug-resistant Pseudomonas aeruginosa.

This article is part of the Topical Collection on Medicine

Veronika Krasnanova

veronika.krasnanova@gmail.com

1 Pediatric Cardiac Intensive Care Unit, National Institute of Cardiovascular Diseases, Limbova 1, Bratislava, Slovakia

\section{Case Presentation}

A 5-month-old boy with patent arterial duct, congestive heart failure, and respiratory syncytial virus infection was admitted to pediatric intensive care unit for respiratory failure. While intubated on mandatory ventilation, the patient developed sepsis and hemodynamic instability requiring inotropic support with catecholamines. Empiric therapy with meropenem, gentamicin, and fluconazole was initiated. To stabilize hemodynamics, transcatheter occlusion of patent arterial duct was performed. Microbial specimens from upper respiratory tract, bronchoalveolar lavage, and urine showed a presence of multi-drug-resistant Pseudomonas aeruginosa. Oropharyngeal swab was taken by inserting swab into the posterior pharynx and tonsillar areas and rubbed over both tonsils and posterior wall of oropharynx while avoiding contact with other areas of the oral cavity. Nasopharyngeal swab was taken by inserting swab through the nares parallel to the palate until resistance was indicating contact with the nasopharynx (approximately the distance equivalent to that from the ear to the nostril of the patient). Then, the swab was rubbed and left in place for several seconds to absorb secretions. Blood cultures remained negative. Based on minimum inhibitory 
concentration, the therapy was changed to amikacin and colistin. Meropenem was discontinued due to resistance. Three days later, the patient clinical conditions deteriorated. Low cardiac output syndrome was treated by combination of epinephrine, norepinephrine, and terlipressin and acute respiratory distress syndrome by high frequency oscillatory ventilation, inhaled nitric oxide, and pulmonary surfactant. In case of serious sepsis and carbapenem resistance, high-dose meropenem $(40 \mathrm{mg} / \mathrm{kg}$ every $8 \mathrm{~h}$ ) was considered but could not be administered because of oligoanuria. Thus, the dose of $20 \mathrm{mg} / \mathrm{kg}$ was given on the first day followed by $10 \mathrm{mg} / \mathrm{kg}$ for the next 28 days. Organ dysfunction involved also other systems, and the patient developed pancytopenia, hepatopathy with cholestasis, paralytic ileus, and renal failure with anuria. Despite combined antibiotic therapy with meropenem, amikacin, and colistin, procalcitonin and Creactive protein increased to $85 \mathrm{ng} / \mathrm{ml}$ and $482 \mathrm{mg} /$, respectively (Fig. 1). Because of life-threatening condition, tigecycline was added to the therapy with permission and agreement of the parents. Tigecycline was administered intravenously twice daily with a loading dose of $2 \mathrm{mg} / \mathrm{kg}$ and then $1 \mathrm{mg} / \mathrm{kg}$. Within $48 \mathrm{~h}$, inflammatory markers started to decrease and cardiac output improved. Tigecycline therapy continued for 13 days without adverse effects. Then, amikacin, colistin, and meropenem were gradually discontinued. Catecholamines were reduced. Renal and hepatic impairment improved. Patient was successfully extubated on the 28th day of hospitalization. Patient fully recovered and was discharged from intensive care unit after 39 days.

\section{Discussion and Conclusions}

In the recent era, raising bacterial resistance to standard antibiotics resulted in limited options for the treatment of serious infections. One of the most common multi-drug-resistant bacteria is a gram-negative non-fermenting bacillus, Pseudomonas aeruginosa, being the major infectious agent responsible for ventilator-associated pneumonia, catheter-associated urinary tract infections, surgical site infections, and intra-abdominal infections [1]. The risk of developing infection with multidrug-resistant pathogens depends on the presence of risk factors (hospitalization longer than 5 days, recent hospitalization within 90 days, recent antibiotic therapy, residence in a nursing home, need for chronic care outside the hospital) [5]. The risk of intrinsic and acquired resistance is an important factor while selecting empiric or directed therapy. Common resistance to standard antibiotics in Pseudomonas aeruginosa infections limits the therapeutic options. The antipseudomonal antibiotic agents include penicillin-beta-lactamase combinations (piperacillin-tazobactam), cephalosporins, monobactams, fluoroquinolones, carbapenems, aminoglycosides, and polymyxins. In severe infections, combination of antibiotics is advocated $[2,6]$. In some cases, antibiotic combinations may include agent, to which bacteria show microbial resistance [7]. Synergistic effects against multi-drug-resistant bacteria, including Pseudomonas aeruginosa, have been revealed for double and triple antibiotic combinations including an aminoglycoside, meropenem, colistin, a fluoroquinolone, a macrolide, or rifampin $[8,9]$. Colistin with meropenem may also be considered salvage therapy for carbapenem-resistant Pseudomonas aeruginosa strains [10]. In our patient case, we presumed that returning meropenem to the therapy would result in synergic effect with amikacin and colistin. However, despite the combination therapy, patient condition deteriorated with worsening of multi-organ failure. Adding a tigecycline into the therapy resulted in inflammatory markers decline and improvement of clinical condition.

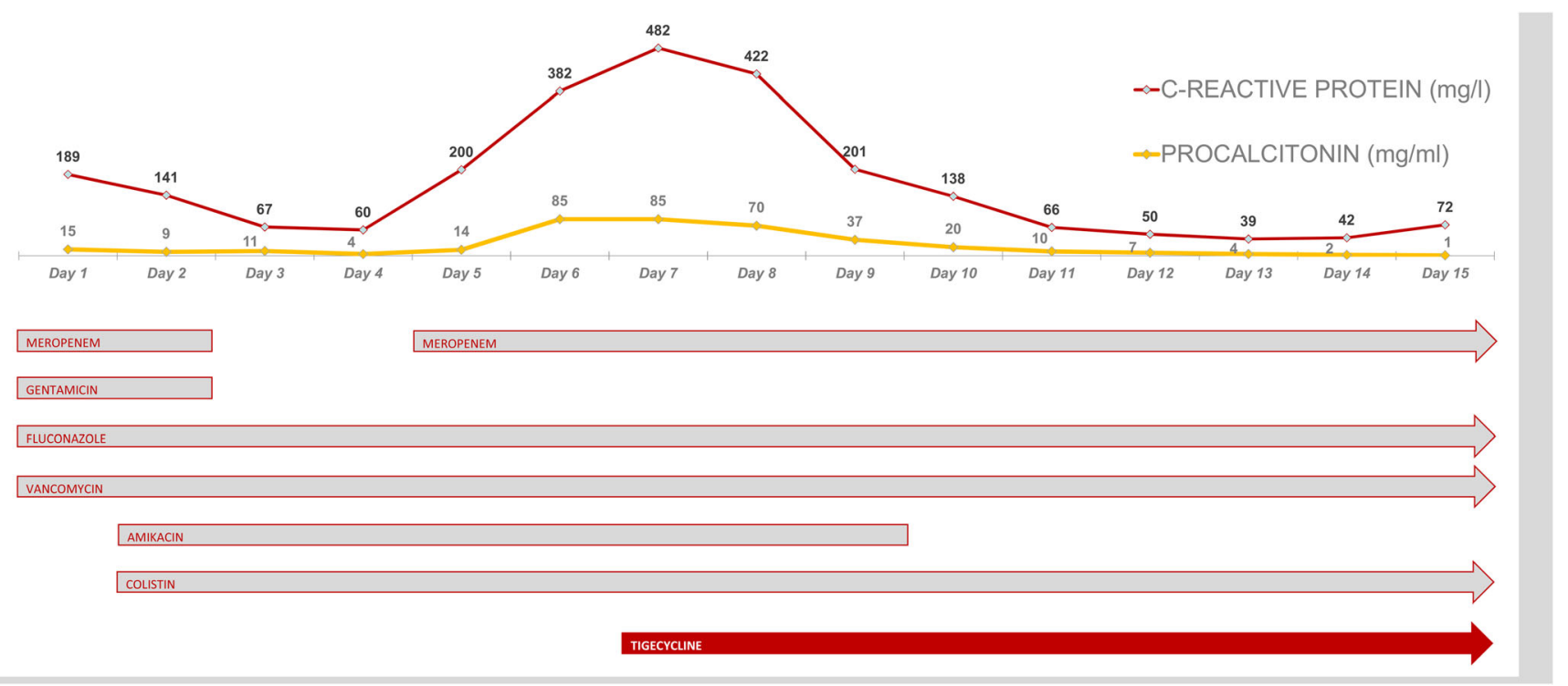

Fig. 1 Inflammatory markers in an infant with multi-organ failure and severe sepsis caused by multi-drug-resistant Pseudomonas aeruginosa 
In adults, there are many clinical trials with the use of tigecycline as a broad-spectrum antibiotic. In infants, only a few cases of tigecycline use have been reported because of side effects that include nausea, vomiting, diarrhea, acute pancreatitis, teeth discoloration, and neutrophil engraftment delay $[3,11]$. However, the recent data show that tigecycline may represent a considerable treatment option in life-threatening infectious complications in pediatric patients as a part of combination therapy. Although Pseudomonas aeruginosa is not reliably inhibited by tigecycline, it seems to be a highly active antimicrobial agent in critically ill and immunocompromised pediatric patients [12].

Iosifidis et al. [13] conducted a retrospective chart review of 13 children treated with tigecycline. The youngest child was 2.5 months old. Tigecycline was used for managing bacteremia, pneumonia, and three unspecified infections. Song et al. [4] described a case series of 22 pediatric patients suffering from serious infections after surgery with median age of 7.5 months who received an initial dose of $1.5 \mathrm{mg} / \mathrm{kg}$ and then $1.2 \mathrm{mg} / \mathrm{kg}$ of tigecycline every $12 \mathrm{~h}$. Median duration of treatment was 17 days and $86 \%$ of patients had positive response. Emiroglu et al. [14] described a 5-month-old infant with ventriculo-peritoneal shunt meningitis. Tigecycline dose of $1.2 \mathrm{mg} / \mathrm{kg}$ twice a day was administered for 20 days. In our patient, tigecycline was used as a salvage therapy in combination with other antipseudomonal antibiotics in a patient with sepsis-induced multi-organ failure. Tigecycline was given for 13 days twice daily with the initial dose of $2 \mathrm{mg} / \mathrm{kg}$ and maintenance dose of $1 \mathrm{mg} / \mathrm{kg}$. We observed no adverse effects during therapy. Tigecycline did not eradicate Pseudomonas aeruginosa, although inflammatory markers decreased which may be raised initially because of other conditions rather than sepsis. Although Pseudomonas aeruginosa colonization persisted, inflammatory markers decreased and patient fully recovered. We can conclude that cardiac failure in an infant with congenital heart disease can be complicated with respiratory failure due to viral infection and, subsequently, with multi-organ failure in case of Pseudomonas sepsis. Based on our patient case, we suggest that tigecycline in combination with other standard antipseudomonal antibiotics may be considered in children for the management of severe multi-drugresistant bacteria infections when conventional treatment has failed.

Acknowledgments The authors are thankful to the team of Pediatric Cardiac Intensive Care Unit in National Institute of Cardiovascular Diseases in Bratislava.

Author Contributions VK and LK cared for the patient, drafted the manuscripts, and carried out the literature research. Both authors read and approved the final manuscript.

Data Availability All data are included in this published article.

\section{Compliance with Ethical Standards}

Ethics Approval and Consent to Participate Written informed consent was obtained from the parents to participate on this study. This study was approved by the Institutional Review Board of the National Institute of Cardiovascular Diseases in Bratislava, Slovakia.

Consent for Publication Formal written informed consent was obtained from the parent for the publication of this case report.

Conflict of Interest The authors declare that they have no conflicts of interest.

\section{References}

1. Lutsar I, Telling K, Metsvaht T. Treatment option for sepsis in children in the era of antibiotic resistance. Expert Rev Anti-Infect Ther. 2014;12(10):1237-52.

2. Du X, Ying F, Yunsong Y. Tigecycline treatment of infection caused by KPC-producing Escherichia coli in a pediatric patient. Ann Clin Microbiol Antimicrob. 2013;12:19.

3. Peng C, Wang X, Zhang J, et al. Tigecycline application in a 3month-old infant with multiple drug resistant Klebsiella pneumonia: a case report. Gut Pathog. 2018;10:25.

4. Song Y, Hua L, Shu Q, et al. Tigecycline salvage therapy for critically ill children with multidrug-resistant/extensively drugresistant infections after surgery. Int J Infect Dis. 2018;75:82-8.

5. Nair GB, Niederman MS. Nosocomial pneumonia: lessons learned. Crit Care Clin. 2013;29(3):521-46.

6. Betts JW, Phee LM, Hornsey M, Woodford N, Wareham DW. In vitro and in vivo activities of tigecycline-colistin combination therapies against carbapenem-resistant Enterobacteriaceae. Antimicrob Agents Chemother. 2014;58(6):3541-6.

7. Taneja N, Kaur H. Insights into newer antimicrobial agents against gram-negative bacteria. Microbiol Insights. 2016;9:9-19.

8. Fish DN, Choi MK, Jung R. Synergic activity of cephalosporins plus fluoroquinolones against Pseudomonas aeruginosa with resistance to one or both drugs. J Antimicrob Chemother. 2002;50(6): 1045-9.

9. Tängdén T. Combination antibiotic therapy for multidrug-resistant gram-negative bacteria. Ups J Med Sci. 2014;119(2):149-53.

10. Sarda C, Fazal F, Rello J. Management of ventilator-associated pneumonia (VAP) caused by resistant gram-negative bacteria: which is the best strategy to treat? Expert Rev Respir Med. 2019;13(8):787-98.

11. Montravers P, Dupont H, Bedos JP, et al. Tigecycline use in critically ill patients: a multicentre prospective observational study in the intensive care setting. Intensive Care Med. 2014;40(7):988-97.

12. Bassetti M, Poulakou G, Giamarellou H. Is there a future for tigecycline? Intensive Care Med. 2014;40:1039-45.

13. Iosifidis E, Violaki A, Michalopoulou E, Volakli E, Diamanti E, Koliouskas D, et al. Use of tigecycline in pediatric patients with infections predominantly due to extensively drug-resistant gramnegative bacteria. J Pediatr Infect Dis Soc. 2017;6(2):123-8.

14. Emiroglu M, Alkan G, Dagi HT. Tigecycline therapy in an infant for ventriculo-peritoneal shunt meningitis. Pediatrics. 2017;139(1).

Publisher's Note Springer Nature remains neutral with regard to jurisdictional claims in published maps and institutional affiliations. 